\title{
Intelligent manipulation control for robotic system
}

\author{
Tsymbal Oleksandr ${ }^{1}$ \\ Mordyk Oleksandr ${ }^{2}$
}

${ }^{1}$ Kharkiv National University of Radio Electronics, Nauki Ave. 14, Kharkiv,61166,Ukraine, oleksandr.tsymbal@nure.ua

${ }^{2}$ Kharkiv National University of Radio Electronics, Nauki Ave. 14, Kharkiv,61166,Ukraine,kurtwalkir@gmail.com

\begin{abstract}
There is provided an analysis in development of flexible integrated systems of industrial application. Report shows an increasing importance of intelligent components for control systems. There are considered the basic items on development of intelligent decision-making support systems to solve the manipulation tasks of industrial robots. The language-oriented constructions for knowledge bases of intelligent problem solvers are presented as well as specifications of their practical implementation and perspective development directions.
\end{abstract}

Keywords: intelligent control; decision-making support; intelligent problem solver; robotics; manipulations.

\section{INTRODUCTION}

The analysis of tendencies of development of flexible integrated production systems indicates the increasing complexity of the organization of modern production, both at a separate workplace and in the conditions of a workstation, workshop or plant. In these circumstances, the role of automated control systems that use AI tools capable to obtain, with good quality, information about the state of production systems, to analyze them, and to make decisions to ensure the functioning of the enterprise, is growing. The role of production decisions in every specific workplace, which becomes a function of servicing equipment: industrial robots, robocars, other providing and technological systems [1, 2], is increasing. At the same time, the problem of the development and implementation of intellectual support for decision-making at different levels of management of flexible integrated production systems remains actual.

\section{BASIC TASKS OF INTELLIGENT CONTROL SYSTEMS FOR MANIPULATIONS}

The plan of decision for manipulation robot is described as a sequence of actions, starting from the beginning, through the transformation of the states of the system - to the achievement of the goal (or goals). Among the implementations of planning systems there should be mentioned such systems as STRIPS, NONLIN, PRODIGY [2]. The planning process is divided into decomposition and coordination phases [3].

The plan development system consists of a general problem solver associated with many training modules; the system also includes the following components: EBL (Explanation-based learning), blocks for defining analogy derivatives, abstraction of plans, and validation. The descriptive language implemented, for example, in PRODIGY (PDL) is a form of predicate logic that supports standard logic operations for multiple sets of elements [4].

In order to describe the plan generator as a tool to process rules and to implement the planning process, and in order to solve concurrently existing tasks (two-handed implementation), [2] proposes language CSA. Here, the rules are described using CSA formulas. The CSA plan generator manipulates pre- and post-conditions. To describe the rules for workspace with three blocks, the pre- and post-conditions $\mathrm{C} 1$ - $\mathrm{C} 4$ are defined as:

$$
\begin{aligned}
& C 1=\operatorname{ONTABLE}(x) \wedge C L E A R(x) \wedge H A N D E M P T Y \\
& C 2=H O L D I N G(x) \\
& C 3=H O L D I N G(x) \wedge C L E A R(y) \\
& C 4=H A N D E M P T Y \wedge C L E A R(x) \wedge O N(x, y)
\end{aligned}
$$

To define all the possible conditions, there are used operators to update the conditions and identity statements for description. The status of the current situation is described by rules such as PICKUP, PUTDOWN, STACK, UNSTACK. The identity statement indicates the conditions for non-compliance. The problem solver consists of working memory, knowledge base (rules, frames, networks) and inference engine [5].

The inference engine creates function of possible actions to the current state, generates new routs. The planning process includes stages of description of current system's state; of search of rules (by inference engine) corresponding to currents state; of finding rules, corresponding to current state for inference engine; of creation (by inference engine) transitions for current state and generation of new system's states.

The further use of strategic planning methods should be based on logical models, considering the dynamic nature of intellectual robotic manipulation systems.

\section{CONCLUSION}

The current problem with modern flexible integrated production systems still remains in a the provision of production functions under the effect of various external factors, including those associated with changes in the conditions of execution of technological operations, adaptation to new conditions of production technology, interaction with other technological equipment. These tasks can be solved by the decision support systems of intelligent control systems, implemented for mobile and manipulation robots.

\section{REFERENCES}

[1] O. Tsymbal, A. Bronnikov, A. Yerokhin. Adaptive Decision-making for Robotic tasks // Proceeding of IEEE 8th Conf. Advanced Optoelectronics and Lasers, CAOL-DSMOLE*2019: "Data Science in Modern Optoelectronics and Laser Engineering", Sept. 6-8, Sozopol, Bulgaria. - P. 594-597.

[2] Xin-She Yang (ed). Artificial Intelligence, Evolutionary Computing and Metaheuristics. Springer, 2013, 796 p.

[3] Zheng C., Liu H., Ge M., Liu Y. A Novel Maze Representation Approach for Finding Filled Path of a Mobile Robot, Int. Conf. Computer, Network, Communication and Information Systems (CNCI 2019), Advances in Computer Science Research, volume 88, pp. 664-673, 2019.

[4] Tsymbal O., Bronnikov A. Decision-making information technology for flexible integrated manufacturing / Modern state of science researches and technologies. 2019. № 2 (8). - C. 105-112.

[5] Bronnikov, I. Nevliudov, O. Tsymbal. Flexible manufacturing tendencies and improvements with visual sensoring / Eskisehir Technical University Journal of Science and Technology. Applied Sciences and Engineering, 2019. Vol. 20, ICONAT issue, P. 77-83. 